\title{
Snakebite in Bobo-Dioulasso, Burkina Faso: illustration of realities and challenges for care based on a clinical case
}

Kyelem CG (1, 2), Yaméogo TM (1, 2), Ouédraogo SM (1, 2), Zoungrana J (1), Poda GEA (1, 2), Rouamba MM (1), Ouangré A (1), Kissou SA (3), Rouamba A (4)

(1) Department of Medicine, Sourô Sanou de Bobo-Dioulasso Teaching Hospital Center (CHUSS), Bobo-Dioulasso, Burkina Faso; (2) Higher institute of Health Sciences, Bobo-Dioulasso, Burkina Faso; (3) Department of Pediatrics, Sourô Sanou de Bobo-Dioulasso Teaching Hospital Center (CHUSS), Bobo-Dioulasso, Burkina Faso; (4) Department of Surgery, Sourô Sanou de Bobo-Dioulasso Teaching Hospital Center (CHUSS), Bobo-Dioulasso, Burkina Faso.

\begin{abstract}
We report herein the case of 19-year-old female farmer who suffered a double snakebite on the right foot. After an unsuccessful traditional treatment, she consulted a health center, 48 hours after the bite. Upon arrival at the hospital, she showed signs of severe damage, including hemorrhagic syndrome, extensive gangrene of the bitten limb and severe acute renal failure. Due to financial constraints, neither antivenom nor the scheduled amputation was performed. After 35 days of hospitalization, she returned home, against the advice of medical personnel. Our case summarizes the daily challenges of patients and practitioners that suffer snakebite envenomation in Bobo-Dioulasso, western Burkina Faso.
\end{abstract}

Key words: envenomation, snakebite, management, Burkina Faso, sub-Saharan Africa.

\section{INTRODUCTION}

Snakebites constitute a serious public health problem that, when neglected, poses serious consequences. The WHO estimated that 5 million snakebites, including 2.5 million envenomations occur in the world annually (1). They are responsible for at least 100,000 deaths and about three times more amputations and other permanent disabilities (2). Snakebite incidence and mortality are underestimated, with less than $20-30 \%$ of actual data being reported in current statistics (3). All sub-Saharan Africa countries are confronted with this problem, both in urban and rural areas $(3,4)$. In Burkina Faso, a landlocked country in West Africa, more than 20,000 cases are reported each year, according to the Ministry of Health (data being analyzed).

Herein, we describe the progression of a typical snakebite case to illustrate the difficulties of managing envenomation in West African countries.

\section{CASE REPORT}

On June 18, 2012 at around 8 a.m., when she was harvesting shea nuts in Kimini, a village $60 \mathrm{~km}$ from Bobo-Dioulasso (the second city of Burkina Faso), Mrs. AB, aged 19, was bitten twice on the right foot. While observing her foot, she noticed bleeding from two points she attributed to snakebite. After ineffective traditional treatment for two days and because of the onset of a large painful swelling of the bitten limb associated with vomiting and hematemesis, Mrs. AB consulted the Center for Health and Social Promotion (CSPS) in Kimini on June 20, where she was referred to CHUSS on June 21. 
No specific treatment was undertaken at CSPS.

Clinical examination noted: large edema of right foot and leg; hemorrhagic syndrome with hematemesis, bleeding from fang marks and scars; poorly tolerated clinical anemia; and oliguria.

Biological tests noted severe hypochromic microcytic anemia (hemoglobin $=3.4 \mathrm{~g} / \mathrm{dL}$ ), thrombocytopenia (platelets $=76,000 / \mathrm{mm}^{3}$ ), predominantly granulocytic leukocytosis (white blood cells: $25600 / \mathrm{mm}^{3}$ ), increasing activated partial thromboplastin time (aPTT) and activated partial thromboplastin time (APTT), hypercreatininemia at $1,082 \mathrm{micromol} / \mathrm{L}$, and blood urea higher than $10 \mathrm{mg} / \mathrm{L}$.

The bite was imputed to Echis ocellatus, due to clinical and biological presentation, size of fang marks and the fact that this species is responsible for about 75\% of envenomations (5).

The treatment consisted of administration of red blood cell infusion, antitetanus serum, hydroelectrolyte rehydration, antibiotics (ceftriaxone and metronidazole), anti-inflammatory (alphaamylase) and local care of the right lower limb. Antivenom was recommended but not administered due to financial inaccessibility for the patient and his family. Similarly, fresh frozen plasma transfusion could not be performed because of the unavailability of this product in the blood bank.

Although the patient recovered from both hemostatic and renal defects, the evolution was marked by the appearance of blisters and secondary signs of gangrene of the right foot, quickly extending to the leg, with the presence of areas of necrosis of the soft tissues of the toes, ankle and lower leg. Daily abrasions and dressings were carried out in collaboration with the Department of Surgery.

Amputation of the right foot was scheduled but postponed initially due to the poor condition of the patient, which was not conducive to surgery. Although scheduled again after her condition had improved, it could not be performed due to the financial indigence of the patient and his family who could not extend the hospitalization stay.

After 35 days of hospitalization, Mrs. AB returned to Kimini on July 27, 2012, against advice of medical personnel.

\section{DISCUSSION}

Mrs. AB's case illustrates the realities and difficulties of snakebite management in Africa, which we would like to highlight.

First of all, the long delaybetween snakebite and presentation (herein, two days before reaching the health center), which impedes clinical outcome, could have two main causes, namely the distance and the scarcity of health centers that do not favor rapid consultation. Furthermore, victims often arrive in health centers after a complex itinerary: first a traditional healer in most cases, then a remote health center where no treatment is available, and finally the reference center, also lacking therapeutic means $(3,6,7)$.

Health personnel, usually a first-line nurse or health worker, is often surprised by the severity and complexity of symptoms, namely severity of local symptoms (edema and gangrene), abundance of hemorrhage, and acute renal failure. In most health centers, a lack of equipment and other resources precludes the treatment of such complications. Moreover, the absence of an antivenom - whose cost is prohibitive - leads to untenable situations for the medical team which is distraught, as well as for the patient and his relatives who consider themselves abandoned by modern medicine. It must be remembered that the early administration of antivenom would reduce mortality by $90 \%$ (6). Mrs. AB's case had required care in an intensive care unit, in addition to the administration of antivenom (before blood transfusion), amputation or disarticulation of the gangrenous limb segment $(8,9)$.

Finally, the act of leaving the hospital against medical advice (an "escape" according to the terminology in Africa) causes deep distress to the medical team which feels sorry for the poor conditions of patients and their choice, and regrets the lack of therapeutic means.

\section{CONCLUSION}

Mrs. AB presented a severe case of envenomation following double snakebites, and showed severe locoregional, hematological and renal complications. Her course in seeking treatment and clinical progression appear to be usual in Burkina Faso, and more generally in subSaharan Africa. 
However, it should be emphasized that the African context is marked by insufficiencies of equipment and other aspects of health centers as well as widespread poverty among its populations, especially in rural areas. Therefore, snakebite management is facing many challenges, involving:

- Bite victims, who are delayed a long time sometimes too long - before arriving at the health center for many complex reasons.

- Health facilities are too few in number, scattered, poorly equipped and lacking resources. Shortage of antivenom is once more emphasized.

- Poor financial means for the implementation of appropriate treatment is a recurrent problem. Neither health centers nor patients receive financial support (social security, insurance, etc.) to contribute to treatment cost, which greatly exceeds the average monthly income of a family in Burkina Faso.

- Health personnel lack the necessary training to use a standardized treatment protocol.

- Managers and health decision-makers neglect this public health problem, most often through ignorance of its importance in terms of incidence and severity (10).

Improvement of snakebite management requires the involvement of all stakeholders: policy makers, civil society, health workers, traditional healers, and the general population. The objective is to improve the management of snakebites, now exceeding 500,000 per year in sub-Saharan Africa, in order to dramatically reduce mortality and disabilities by $90 \%$.

\section{COPYRIGHT}

(c) CEVAP 2012

\section{SUBMISSION STATUS}

Received: September 28, 2012.

Accepted: November 6, 2012.

Abstract published online: November 9, 2012.

Full paper published online: November 30, 2012.

\section{FINANCIAL SOURCE}

Centre Hospitalier Universitaire Sourô Sanou de Bobo-Dioulasso provided the financial grants.

\section{CONSENT}

Informed consent was obtained from the patient for publication of this case report.

\section{CORRESPONDENCE TO}

Carole G. Kyelem, 01 BP 676 CHUSS BoboDioulasso. Phone: +226 7011 9432. Email: pasismama@hotmail.com.

\section{REFERENCES}

1. Chippaux JP. Snake-bites: appraisal of the global situation. Bull World Health Organ. 1998;76(5):51524.

2. Organisation Mondiale de la Santé. Aide-mémoire $\mathrm{N}^{\circ} 337.2010$

3. Chippaux JP. Estimate of the burden of snakebites in sub-Saharan Africa: A meta-analytic approach. Toxicon 2011;57(4):586-99

4. Drabo YJ, Sawadogo S, Kaboré J, Chabrier J, Traoré R, Ouédraogo C. Morsures de serpents à Ouagadougou. Aspects épidémiologiques, cliniques, thérapeutiques et évolutifs, à propos de 70 cas. Sem Hop (Paris). 1996;72:849-55.

5. Chippaux JP. Snakebite in Africa: current situation and urgent needs. In: Mackessy SP, editor. Reptile venoms and toxins. Boca Raton: Taylor \& Francis/CRC Press; 2009. p. 445-65.

6. Chippaux, JP. The treatment of snake bites: analysis of requirements and assessment of therapeutic efficacy in tropical Africa. In Ménez A, editor. Perspectives in Molecular Toxinology. New York: John Wiley \& Sons, Ltd; 2002. p. 457-72.

7. Somé N, Poda JN, Guissou IP. Epidémiologie et prise en charge des envenimations ophidiennes dans le district sanitaire de Dano, province du Ioba (Burkina Faso) de 1981 à 2000. Bull Soc Pathol Exot. 2002;95(3):163-6.

8. Bellefleur JP, Le Dantec P. Prise en charge hospitalière des morsures de serpent en Afrique. Bull Soc Pathol Exot. 2005;98(4):273-6.

9. Abubakar SB, Habib AG, Mathew J. Amputation and disability following snakebite in Nigeria. Trop Doct. 2010;40(2):114-6.

10. Chippaux JP. Estimating the global burden of snakebite can help to improve management. PLoS Med. 2008;5(11):e221.

\section{CONFLICTS OF INTEREST}

The authors declare no conflicts of interest. 